\title{
L'espace problématique de la violence politique des enfants
}

Daniel Hermant

\section{(2) OpenEdition}

1 Journals

\section{Édition électronique}

URL : http://journals.openedition.org/conflits/461

DOI : $10.4000 /$ conflits.461

ISSN : $1777-5345$

Éditeur :

CCLS - Centre d'études sur les conflits lilberté et sécurité, L'Harmattan

\section{Édition imprimée}

Date de publication : 15 juillet 1995

ISSN : 1157-996X

Référence électronique

Daniel Hermant, "L'espace problématique de la violence politique des enfants », Cultures \& Conflits [En ligne], 18 | été 1995, mis en ligne le 04 mars 2005, consulté le 30 mars 2021. URL : http://

journals.openedition.org/conflits/461 ; DOI : https://doi.org/10.4000/conflits.461

Ce document a été généré automatiquement le 30 mars 2021.

Creative Commons License 


\title{
L'espace problématique de la violence politique des enfants
}

\author{
Daniel Hermant
}

1 Tués, blessés, déplacés, traumatisés, manipulés ou pervertis, pour tout dire violentés dans leur corps et dans leur esprit, les enfants apparaissent le plus souvent de nos jours comme des victimes. A en croire l'actualité la situation est particulièrement choquante dans les conflits et les guerres extra-européennes, ce qui a amené l'ONU à élargir au monde entier le champ d'activité de l'UNICEF ${ }^{1}$. Actuellement sur tous les continents elle protège l'enfant en le nourrissant, en le soustrayant aux conflits, mais aussi en réaffirmant son droit à l'éducation et en dénonçant les embrigadements forcés, sexuels, laborieux, ou militants - dont il est l'objet. L'institutionnalisation au Nord de ce réflexe humanitaire pour répondre aux violences du Sud, a nourri un discours où parler de la violence politique des enfants dans le tiers-monde, revenait à dénoncer la transgression d'une norme juridique - celle des droits de l'enfant -, comme si la variable âge, qui en amont sépare l'enfance de l'âge adulte, déterminait un seuil d'illégitimité à ne pas franchir ${ }^{2}$. Ce discours a ses références historiques comme le montre a contrario l'embrigadement cynique d'enfants dans les organisations de jeunesses nazies ou fascistes. La violence des enfants, sous-produit, et sous-partie, du thème plus vaste de la violence politique, sert alors de critère pour enregistrer une étape supplémentaire du dérapage de ces sociétés, voire de leur naufrage, elle permet de diagnostiquer une perte de repère, conduisant à une dérive autoritaire, ou/et à une situation d'anomie, ce qui redouble sur un autre plan la condamnation morale à laquelle aboutissait l'approche juridique. A travers les domaines bien balisés du juridique et du politique, la violence enfantine s'inscrit en priorité pour nos contemporains dans la constellation vague de l'humanitaire (et de sa transgression). Cependant cette attitude mélange des réalités bien diverses. Nous évoquions la manipulation politique des enfants sur le sort desquels on s'indigne. Si l'explication est avérée pour les bassidji iraniens ${ }^{3}$ ou des gardes rouges chinois ${ }^{4}$, elle semble bien moins assurée pour les jeunes palestiniens de l'intifada ${ }^{5} \mathrm{Et}$ que dire de la Marseillaise de Rude dont le groupe martial, rappelons-le, comprend un jeune garçon d'une dizaine d'années, ou encore pour les Gavroches ${ }^{6}$ ou les enfants communards du XIXe? A bien y réfléchir, l'enfant victime n'est guère séparable de 
l'enfant bourreau comme en témoignent bien des conflits du tiers monde ou du monde développé, mais également, quoique d'une manière un peu différente, nos villes ou nos banlieues à travers les violences des casseurs et autres émeutiers du week-end. C'est ce qui explique qu'un second discours sur l'enfance, dénonçant les nouveaux barbares qui montent à l'assaut de notre tranquillité, s'entortille autour du précédent et quelquefois l'occulte.

Quelle enfance?

2 Mais d'abord qu'est-ce que l'enfance, ou la jeunesse, car nous avons jusqu'à présent employé les deux termes indifféremment? La notion n'est claire, ni dans le contenu ni dans les limites, car il serait naïf de considérer qu'elle est le produit «naturel» de l'ordre des choses. La biologie fixe moins une frontière assignable à un âge précis, qu'elle ne désigne, avec la notion ambiguë et transitionnelle d'adolescence, un passage progressif où l'âge adulte naît de la jeunesse sans coupure ni interruption ${ }^{7}$. Comme de surcroît les séquences psychologiques qui accompagnent l'évolution physique ont leur rythme propre, on voit bien combien, une fois souligné le rapport direct de l'enfance avec la variable âge, il est difficile d'en tirer des enseignements : plus on tente de préciser le trait, plus la notion se brouille. En fait les connaissances scientifiques accumulées (biologie, médecine, psychologie, pédagogie, démographie...) ne forment pas un socle objectif sur lequel s'installerait, convenablement doté, la notion de jeunesse, mais renvoient à des champs épistémologiques différents qui ne se mettent pas spontanément en série, et qui loin d'être subsumables par la notion de jeunesse (ou d'enfance), la ferait plutôt éclater ${ }^{8}$. La jeunesse telle que nous l'avons entendue dans ce numéro de Cultures et Conflits renvoie à la démarche fondatrice qui en séparant en deux parties une trajectoire biologique, trace une frontière entre deux mondes celui des adultes et celui de l'enfance. Le terme renvoie en priorité au principe de filiation qui définit l'enfant sur la base de sa relation avec ses géniteurs. Aussi notre définition de l'enfance est indépendante d'un critère précis et discriminant d'âge, nos contributeurs ont retenu pour leurs analyses aussi bien l'enfant, le pré adolescent, que l'adolescent ${ }^{9}$. Ainsi définie l'enfance se dessine en creux comme l'inverse du monde adulte; l'enfant est celui qui n'a pas la force physique ou la puissance sexuelle de l'adulte, il est celui qui est dépendant économiquement, qui est nourri, il est celui qui ne sait pas. Mais toute relation négative gagnant en substance quand elle est retournée, la jeunesse est également présentée comme dotée de qualités particulières, différentes, ou supérieures de celles de l'adulte: biologiques avec le trop plein d'énergie et sa violence, psychologiques avec l'instabilité, intellectuelles avec les facultés d'apprentissage et d'adaptation. Enfin les traits caractéristiques de la jeunesse sont, évolutifs, dynamiques, provisoires. Regarder un enfant c'est voir un futur adulte ; c'est envisager sa disparition/transformation. L'opposition enfance/adulte ne distingue pas deux groupes sociaux, doté de cohésion et susceptibles d'être des acteurs, mais sépare deux catégories générales cohérentes par leur principe constitutif et susceptible d'agréger un certain nombre de qualités. Nous sommes en face d'une opposition binaire qui définit un système élémentaire recoupant le sens commun, un peu analogue à la division par sexes. L'enfance renvoie à la thématique immémoriale des âges de la vie et s'inscrit comme catégorie dans le long terme par un contenu qui agrège de multiples visions de l'enfant (ou de l'enfance, ici les deux termes sont synonymes) qu'on peut classer autour de deux pôles: vieux pessimisme des moralistes avec ses jugements négatifs, ou inversement, optimisme de la longue tradition pédagogique et affective. Perçue comme catégorie l'enfance ne renvoie à aucun sujet précis mais les accueille 
tous, c'est un lieu commun qui alimente par des exempla - ou des imago - n'importe quelle réflexion sur l'éducation et les moeurs ${ }^{10}$. Elle se situe dans le champ du raisonnement rhétorique, pas dans celui de l'analyse sociale.

Une matrice : Deux enfances

3 Pour revenir à celle-ci, il faut souligner que la violence politique enfantine ne peut exister que si l'enfant est acteur politique et l'enfance «concept politique». Les travaux classiques de $\mathrm{Ph}$. Aries ${ }^{11}$ établissent que la notion d'enfance a été inventé à l'âge moderne. Avant, si les enfants existaient, l'enfance n'existait pas : «L'enfance est la vie d'une bête » disait Bossuet largement représentatif en cela de l'esprit classique. A la fin du XVIIIe siècle les perceptions changèrent ; une révolution de la sensibilité dont témoigne, entre autres, le succès de l'Emile de Rousseau, substitua au petit être cruel, illogique et moins rationnel qu'un animal, que dépeignait La Fontaine, un enfant innocent, sensible, à protéger et à aimer. Ce nouveau regard se porta également sur la place et le rôle de la jeunesse, en tant que groupe particulier, dans la société. L'émotion puis les réflexions qu'entraînèrent le travail forcé des enfants au XIXe siècle, mirent au premier plan le rôle social et économique de l'enfant. Ce n'est pas une mais deux enfances qui émergèrent de ces transformations. Le bon enfant ou la belle jeunesse celle qui est éduquée - remarque Frédéric Chauvaud s'oppose à la jeunesse violente et sans doute délinquante qu'on trouve sur les barricades jusqu'à la Commune. Opposée mais hiérarchisée, l'enfance livrée à elle-même, incontrôlable, brouillonne, violente et perverse, est le passé de la société, la jeunesse soumise aux adultes, innocente et studieuse, son avenir. Aussi un long combat humanitaire et politique, libéra-t-il l'enfant des servitudes du travail et l'installa au centre d'un espace pacifié où enfin il put être éduqué $^{12}$. Au XIXe la jeunesse est passée du statut de catégorie universelle à celui de groupe ayant une place, un poids et un coût dans la société. Cette transformation de l'enfance et de la jeunesse a des conséquences directes sur la notion de violence politique enfantine. Examinées du côté de la finalité sociale nos deux jeunesses s'opposent. L'enfance violente signifie la rupture entre les générations, l'opposition entre adultes et enfants. On peut mesurer la place de cette violence. Elle demeure limitée, à la fois disséminée dans des lieux particuliers et monopolisée par des groupes spécifiques, en tout cas minoritaire par rapport à l'action des autres acteurs violents. On peut aussi en recenser les formes. Le plus souvent il s'agit de violences « anthropologiques " liées, dans une société régulée, à l'insertion des jeunes dans le monde adulte: rites de passages, concurrence entre jeunesses de villages dont témoigne abondamment la littérature ethnographique du XIXe siècle, mais dont on pourrait retrouver l'équivalent dans les quartiers défavorisés du XXe. Dans d'autres cas quand le lien social se desserre ou se rompt, (1789-1799 en France, 1917-1921 en Russie, et aujourd'hui : conflits internes ou guerres du tiers monde), des groupes entiers de jeunes tombent dans la violence: violence individuelle des enfants abandonnés, violence collective de groupes organisés pour survivre par la prédation, violence directement politique d'enfants socialisés par la guerre. Au XIXe cette situation a pu être ressentie par les contemporains dans un contexte de guerre sociale, d'une manière extrême ${ }^{13}$; au XXe siècle dans les métropoles des pays développés, la violence de la jeunesse - surtout de la jeunesse exotique - de nos banlieues, fait plutôt l'objet de réflexions désabusées. L'enfance studieuse, majoritaire elle, souligne par contraste le passage de témoin, l'emboîtement harmonieux des deux âges. L'enfant qui incrémente la société ne peut ni en être séparé, ni s'y opposer. En utilisant de préférence à la notion "oppositionnelle» de génération, celle plus transitive de classe d'âge ${ }^{14}$, elle 
envisage le passage de l'une à l'autre comme relevant d'une évolution fluide dominée par l'apprentissage ou l'éducation. C'est moins une frontière qui les sépare qu'une membrane à travers laquelle l'équilibre des deux milieux s'établit progressivement par imprégnation, de l'adulte vers l'enfant, car les liens sont étroitement vectorisés. L'éducation se réduit à un apprentissage de contenu ou à l'intériorisation de pratiques ne laissant aucune autonomie à l'enfant. De cela la pédagogie, partie la plus contrôlée du processus de socialisation des individus, est le témoin. Sur le plan de l'individu elle ne peut imaginer l'enfant autrement que comme une esquisse ou une réduction du futur adulte. Au niveau collectif, à en croire Durkheim, elle n'est efficace que parce qu'elle exprime le lien fondamental de l'enfant à la collectivité. Aborder le groupe par le filtre de l'éducation individuelle privilégie une lecture conservatrice qui en supprimant toute autonomie à l'enfant l'empêche d'être un véritable acteur politique. Le moule éducationnel ne laisse donc que peu de place à la violence, à moins de prendre ce mot dans le sens de contrainte. Durkheim voyait dans la discipline la règle primordiale de la pédagogie car il la jugeait indispensable pour construire une personnalité stable ${ }^{15}$. L'éducation était pour lui contrôles pulsionnels, apprentissage de la maîtrise des passions. En généralisant on peut dire que la perpective psychologique et individuelle dans laquelle l'éducation considère la violence, la réduit à un comportement déviant redevable de techniques psychologiques ou médicales, ou encore de « de la verge et de la férule » comme disait La Bruyère ${ }^{16}$. Le corollaire de cette conception est que le politique - et plus encore la violence politique définie comme autonomie décisionnelle d'un individu ou d'un groupe - est refusé à l'enfant. Annick Percheron a insisté avec force sur le tabou de la politique dans le cadre banal de l'école, mais la remarque doit être élargie car l'enjeu dépasse l'institution scolaire. L'enfance est donc posée comme devant être hors du champ politique et son inclusion, quand elle se produit, est présentée comme transgressive.

Les voies du discours : imitation manipulation et indignation

4 Nous pouvons maintenant revenir aux discours sur la violence des enfants que nous évoquions au début de ce texte. Examinés dans leurs finalités et malgré la grande diversité des situations dont ils rendent compte, ces discours, enserrés dans ce qu'on peut considérer comme une matrice implicite dont ils doivent satisfaire les réquisits, n'interrogent pas les particularités ou les spécificités, mais à l'inverse fonctionnent comme opérateur d'unité. Ils ne renvoient pas une démarche de type analytique ou réflexif, mais illustrent plutôt une démarche de nature rhétorique unifiant la réalité autour de trois éléments en quelque sorte " précontraints ». Le premier d'entre eux est la similitude enfant/adulte. Nous avons noté l'absence d'autonomie de l'enfant qui inscrit l'enfance dans un espace anonyme, ou mieux qui le situe dans un lieu commun. Nous avons également noté sa conformité avec le modèle adulte. L'inclusion de l'enfant, ainsi défini, dans la sphère de la violence politique ne peut se faire qu'à l'initiative des adultes et dans le but de faciliter l'imitation. Les régimes autoritaires ont institutionnalisé ces pratiques : des jeunesses hitlériennes aux balillas, en passant par les jeunesses communistes les exemples historiques sont nombreux. Ici même J. Andrieu nous a rappelé le cas des gardes rouges répondant à l'appel de Mao Zedong, F. Khoroskhavar a analysé le rôle du pouvoir dans la mobilisation des bassidji. Mais les démocraties présentent un cas de figure analogue comme le montre l'analyse de la place des enfants en France, ou en Angleterre, pendant la guerre 14/18 ${ }^{17}$. L'idée qu'il était indispensable de faire partager les objectifs et la légitimité de la guerre aux enfants, même très jeunes, de façon à ne pas les couper de leurs pères combattants et 
de les rendre dignes des sacrifices consentis s'est rapidement imposée à tous et a été prise en charge par l'institution scolaire, avant d'être largement diffusée par la presse et la littérature pour enfants. Ce contexte d'admiration juvénile pour les pères combattants, explique également qu'un certain nombre d'enfants ait souhaité devancer l'âge du service militaire, et se soit même engagé en fraudant sur leur âge. Cet héroïsme de bon aloi montre symboliquement le passage de témoin : la jeunesse partageant déjà les valeurs des aînés, est prête à partir pour le front et à s'agréger par le sacrifice à la communauté des adultes. On peut retrouver chez les gardes rouges ou chez les bassidji la même volonté pure et idéaliste de reproduire à l'identique la société et de défendre le régime. Dans le rapport à la guerre l'enfance prépare l'âge adulte, s'y articule ou s'y emboîte harmonieusement. Le bas relief de Rude $^{18}$ en fournit l'image symbolique puisque métaphore de la défense de la patrie, c'est aussi une métonymie de la mobilisation de tous, y compris des enfants. Sur l'idée d'imitation se greffe celle de la manipulation que les adultes font subir aux enfants. Ils enrégimentent la jeunesse et l'introduisent dans le monde politique de force, c'est à dire sans lui donner le temps ni les moyens de prendre une distance critique. Cet endoctrinement ordinaire dans tous les régimes autoritaires, renforce les commentaires désabusés ou indignés sur la violence des (faite aux) enfants. La notion de manipulation que les articles de ce numéro ont discuté peut maintenant être précisée. Manipulation et imitation ne sont que deux manières de qualifier un rapport entre le monde des adultes et celui de l'enfance dans lequel l'action politique reste un privilège des adultes. Ainsi l'inclusion individuelle des enfants dans le monde de la violence politique peut être interprétée comme imitation glorieuse ou comme transgression insupportable : question de point de vue de l'observateur, mais dans les deux cas elle repose sur l'idée commune que l'enfant appartient à la société, et que celle-ci est contrôlée par l'adulte. Présentée comme particulièrement cynique la manipulation n'est pourtant que l'application à une catégorie particulière de l'attitude "utilitariste» de la plupart des dirigeants politiques à l'égard de ceux qui dépendent d'eux. De ce point de vue la situation enfantine de docilité totale et d'engagement désintéressé fait de l'enfant le meilleur symbole de l'obéissance à l'Etat. La leçon pourrait être retournée et les adultes être invités à imiter des enfants si purement engagés qu'ils n'ont comme projet que ceux du pouvoir politique! L'indignation est le dernier lieu commun de notre série et également le plus englobant comme l'indique la prolifération des discours sur l'enfance victime. Ce sentiment s'inscrit paradoxalement dans la transformation de l'enfance en catégorie "sociale ", c'est à dire dans le dépassement "moderne» de l'analyse k. La construction parallèle de deux enfances au XIXe ne les avait pas placées, nous l'avons $\mathrm{vu}$, sur un pied d'égalité mais les avait hiérarchisées en les inscrivant dans une dynamique. L'attitude de l'UNICEF tentant par le biais de la protection juridique d'étendre le statut de notre jeunesse éduquée à la jeunesse martyrisée des pays du tiers monde, s'inscrit très exactement dans cette perspective et projette dans l'espace actuel le clivage originel du XIXe (mais en substituant à l'écart temporel la distance géographique). Cette dynamique excluait la violence de l'horizon de l'enfance ${ }^{19}$ ou plutôt la réduisait, parce qu'elle existe tout de même, à des dérapages d'adultes, ou aux actes d'enfants pervertis, donc la situait sur un registre relevant de l'indignation ou du compationnel. La plasticité de la notion de victime transformant par un petit effort dialectique les enfants-bourreaux en ramification, fâcheuse et ultime, du rameau des victimes, autorisait un réflexe d'indignation générale qui donnait un faux-semblant d'unité au thème de la violence des enfants, mais en même temps dissimulait le fait 
gênant de l'autonomie violente de certains enfants, point central de ce numéro de Cultures et conflits. Il est donc nécessaire maintenant que nous avons percé la pellicule discursive qui entoure notre thème et mis en évidence le verrou interprétatif implicite qu'elle imposait, de reprendre l'analyse sur d'autres bases.

Quel espace pour la violence des enfants : violence politique...

Louis-Jean Duclos ${ }^{20}$ examinant les conditions minimales d'exercice de la violence par les enfants déclare qu'il faut que ceux-ci soient autonomes et capables de discernement. On peut baliser plus fortement cet espace théorique dans la trajectoire infantile à l'aide des catégorisations psychologiques générales de Piaget qui place vers - dix onze ans l'âge où les sociétés d'enfants prennent leur essor, où l'élargissement du rapport de l'enfant à la société permet à la fois une prise de distance permise par le jugement et une insertion par l'action sur la scène politique ${ }^{21}$. Sur ces bases il est possible d'esquisser une sociologie de la violence enfantine. Tout nos auteurs insistent sur le rôle de l'Etat non comme adversaire, mais comme promoteur de la violence juvénile. La visibilité politique de la violence est directement liée au rôle qui lui est attribué dans les luttes de pouvoirs. Rôle capital et visibilité maximale en Chine avec les gardes rouges, en Iran avec les enfants martyrs et dans les territoires occupés, avec la génération de l'intifada où la cause quasi-étatique défendue, place la violence enfantine au coeur du problème palestinien et transforme en héros les enfants. Au Nigeria le même schéma peut être appliqué mais avec des enjeux plus réduits, provinciaux ou communautaires. A contrario l'autonomie relative de la violence des banlieues reçoit son sens de l'extérieur, «négativement » comme le remarque Angelina Peralva. Située à l'écart du politique et du social la violence devient "introuvable " ${ }^{22}$ et est sentie comme anomique. Sur le plan de l'action, les enfants jouent une partition au sein de luttes politiques qui les dépasse. Insérés directement dans la structure du pouvoir, leur violence devient pour partie celle de l'Etat, ou de forces d'oppositions assez conséquentes pour mobiliser la jeunesse C'est le réflexe janissaire utilisé par la Renamo au Mozambique ${ }^{23}$, ce sont les grandes mobilisations organisées par Mao pour séduire les gardes rouges, ou en Iran le rôle des "associations islamiques " qui contrôlent et recrutent les lycéens et celui de Bassidji, qui les envoie au front. C'est au Nigeria les actions des comités d'étudiants ${ }^{24}$ engagés dans les luttes «civiles» ou « communautaires » dont $G$. Nicolas nous dit qu'ils sont considérés comme des vecteurs naturels de la classe politique. Dans tous ces cas le pouvoir politique désigne des cibles : armée régulière (ou non), forces de l'ordre, groupes d'opposants politiques, communautés rivales, boucs émissaires, et met à disposition un cadre organisationnel. L'appui de l'Etat aux groupes de jeunes qu'il mobilise, leur ouvre un espace de pouvoir et de violence très grand ; le pouvoir d'intimidation des Associations islamiques envers les enseignants et l'administration vient en grande partie des rapports que ces jeunes gens entretiennent avec la police secrète, et qu'auraient fait les gardes rouges sans l'armée et l'effet Mao! Ceci n'efface pas pour autant les intentions utilitaristes des gouvernants comme le montre bien la triste histoire des gardes rouges ${ }^{25}$, ou l'envoi cynique d'enfants ou d'adolescents iraniens à la mort dans le cadre de Bassidje. Pourtant la violence peut tout aussi valablement être considérée comme contestataire, provocatrice, enracinée dans un vigoureux réflexe anti-adulte. Venue de la base et plus ou moins spontanée, elle est alors le fait d'individus isolés, de petits groupes ou de foules dans les émeutes ${ }^{26}$. En réalité l'inventaire des actions en aval, ou en amont celui des formes d'organisations, même limité aux mobilisations contestataires, se perd dans les sinuosités du réel tant il renvoie à des situations et à des théâtres variés. Ces 
difficultés de la description proviennent des limites du modèle explicatif étatique qui considère la violence contestataire des jeunes comme périphérique, un peu comme si le sens de la violence s'exténuait quand on passait du monde des rapports de forces à celui des comportements, ou pour reprendre le vocabulaire de L. Coser ${ }^{27}$, des conflits réalistes aux conflits irréalistes. Dans cette perspective les mobilisations ritualisées de l'intifada ou des émeutes de banlieues ont peu à voir avec les manipulations préorganisées de gardes rouges ou de Bassidji, car le profil jeune qui leur est commun n'est considéré que comme une pellicule comportementale anecdotique. Or justement une des originalités de notre numéro a été d'insister sur l'emboîtement des deux dimensions. Le pidou chinois que J. Andrieu décrit longuement, montre bien que l'insertion des gardes rouges dans le projet politique de Mao, n'enlève rien à leur autonomie ; mieux il indique que paradoxalement plus le rôle de l'Etat est fort dans la mobilisation des enfants, plus la marge d'autonomie accordée est elle-même importante et la violence enfantine facilitée. Cela signifie que nous ne sommes que très partiellement en face d'une sociologie du pouvoir et que l'hypothèse selon laquelle la violence politique des enfants définirait un espace politique cohérent appuyé sur des acteurs stables n'est confirmée que partiellement.

...ou symptôme de crise

6 Si le sens des comportements violents s'exténue dans le champ politique, c'est parce que ceux-ci renvoient à des attitudes bien plus complexes que celles du «choix rationnel » ou de la «manipulation politique». Quand on est jeune on se mobilise pour autre chose que des « idées » ou des « concepts » surtout quand ceux-ci sont empruntés au discours des adultes. On se mobilise par goût de l'action, du pouvoir, par plaisir, par imitation, par refus. La violence représente pour les jeunes le charme du passage à l'acte. Loin d'être simplement le reflet d'une impulsion extérieure, les conduites violentes se situent dans un espace d'inventivité sociale où le rapport fort de la violence avec l'image et l'émotion crée de la solidarité, ou de la complicité. Lever certaines contraintes institutionnelles, transgresser l'ordre social, mettre en scène son pouvoir, ou simplement défier l'autorité, fournit de bien grandes satisfactions psychologiques et soude un groupe d'adolescents. Ainsi peinte, cette sociologie est agile, mobile ${ }^{28}$, ludique. Ludique mais aussi sadique, car la férocité - très appréciée au demeurant par certains entrepreneurs de violence - souligne la prise en compte insuffisante de la mort par la jeunesse et l'absence complète d'inhibition sociale liée à son absence de maturité, bref l'inscrit directement dans le champ de l'explication psychanalytique, ajoutons-y sur le plan sociologique, la dynamique collective combinée à la liberté d'action de groupes installés dans les fractures de la société29. Mais la violence infantile est aussi vécue comme un moyen de progresser dans l'échelle sociale, de changer de "condition ». Elle ouvre des opportunités de carrière. Le Mozambique, où certains jeunes kidnappés se transforment en profiteurs de la guerre en obtenant un «statut de guerrier », est l'aboutissement extrême de cette logique, presque un « idéaltype ». L'intérêt individuel est souvent combiné à l'intérêt collectif. En Iran F. Khoroskhavar nous montre comment, pour une partie de la jeunesse, carrière et utilité sociale se mêlent, même si cette dernière se limite au savoir expéditif de l'épuration politique. Considérer la violence politique comme une des modalités d'action de la jeunesse, ainsi que nous venons de le faire, l'a installée au bout d'une seconde chaîne de déterminants, non pas symétrique mais sécante par rapport à celle du pouvoir qui n'y voyait qu'une modalité accessoire de l'action politique. Dès lors le sens de la violence n'est plus à chercher dans un quelconque projet politique, mais à analyser à travers une 
grille sociologique qui la moule de l'intérieur. Notre numéro souligne l'importance des blocages sociaux dans le déclenchement et la permanence de la violence juvénile, il dit que la violence des enfants est liée à l'éclatement des modèles d'organisation traditionnels et peut être interprétée comme une tentative brouillonne, ou désespérée, de socialisation du groupe enfantin. C'est le cas dans la société mozambicaine déstabilisée par l'exode rural et la guerre où les jeunes ne s'inscrivent plus dans une trajectoire claire, c'est le cas en Iran et en Chine où l'essoufflement de la révolution se marque par le conformisme et l'étroitesse d'horizon. A un moindre degré la même observation peut être faite au Nigeria. Dans les banlieues qu'étudie A. Peralva c'est plutôt le chômage ou l'effritement du moule culturel et sociétal unique, une conjoncture bloquée donc, qui favorise l'apparition de la violence. Symptôme de crise, voire indice de décomposition sociale et politique, dont on pourrait préciser les caractéristiques en suivant les trajectoires individuelles dans le détail, la violence comporte enfin une dimension anthropologique qui, bien sûr, conserve son autonomie par rapport à la conjoncture économique et sociale mais la renforce. Camille Mansour montre bien que l'entrée en violence des jeunes palestiniens est liée à la démission des pères devant l'armée israélienne et à la dégradation du modèle palestinien de famille autoritaire qui en est la conséquence, mais la remarque peut être généralisée. Quand le père est martyrisé (Chine), humilié (Palestine) ou simplement déconsidéré (banlieues), c'est tout le mécanisme de reproduction sociale qui est mis en cause !

Conclusion

7 Si les arabesques que dessine la violence sont si difficiles à décrypter, c'est d'abord parce que leurs dessins renvoient à des référents multiples: l'Etat, les groupes de jeunes et les jeunes pris comme groupe, ou plutôt comme catégorie transitoire s'évanouissant dans l'âge adulte. La violence issue des projets ou des stratégies d'acteurs s'inscrit dans l'espace classique et parfaitement lisible de transactions politico-sociales. Les configurations victimaires que dénoncent les discours que nous signalions au début de ce texte, en sont l'illustration typique. Elles voisinent avec des situations inverses dans lesquelles l'enfant prend l'initiative de la violence. Notre numéro s'est surtout attardé sur les configurations mixtes où les deux logiques confluent. Mais la violence renvoie également aux comportements des enfants, à leur "style ». Liée à la psychologie des jeunes, à leur désir d'absolu, d'idéal, de sacrifice, elle correspond alors à une variable secondaire. Enfin sur le plan sociologique, ou anthropologique, la violence est réduite à un simple effet de sens greffé sur des actes ou des trajectoires d'acteurs. C'est dire qu'elle se présente comme un phénomène hétérogène dont l'analyse souligne la dimension kaléidoscopique. Mais il existe une seconde difficulté: en aval les différents niveaux que l'analyse distinguait s'enchevêtrent. Ainsi en Iran, le constat par les jeunes de la rupture de l'unité de la société, leur volonté de la recréer par l'engagement ou par le martyre, mais également le message du guide et les stratégies cyniques du pouvoir, se mélangent et renvoient aux calculs des acteurs et à leurs rêves. Dans ces conditions l'observateur a du mal à évaluer le coût social d'une violence - provoquée, acceptée, subie -, à en peser les effets immédiats ou à moyen terme: comment juger des trajectoires d'enfants ou d'adolescents qui, porteuses d'espoirs au début, se terminent dans les trompe-l'oeil, les faux-semblants, ou, pour les martyropathes, par la mort. Tout se passe pour l'observateur comme si visibilité sociale et attribution du sens étaient inversés : plus la violence est visible, plus son sens devient énigmatique. On ne s'étonnera pas dans ses conditions que les discours aient, la plupart du temps, rabattu l'explication de la 
violence sur la plus classique des causalité : la manipulation par les adultes, et qu'à l'inverse, nous ayons privilégié dans ce numéro les études de cas qui montraient la complexité du phénomène. Face à cette double difficulté il faut convenir que, beaucoup plus qu'à une sociologie classique reposant sur des acteurs stables et des projets identifiables, la violence politique des jeunes renvoit à une phénoménologie dont le dénominateur commun pourrait correspondre à cette "air de famille» dont parle Wittgenstein à propos des jeux ${ }^{30}$. Ténu et transformatif, cet instrument d'analyse permet à partir de la notion de ressemblance de découper facilement l'espace de la violence juvénile dans le tissu social sans avoir à fournir une définition. Dans ce cadre la forte visibilité et l'aspect énigmatique que nous avons relevés provient, à notre sens, de l'entrecroisement inévitable de la violence juvénile avec la notion de jeunesse considérée, cette fois-ci, uniquement à travers sa fonction de reproduction de la société, c'est à dire comme catégorie englobante et structurante ${ }^{31}$. La plupart des articles de ce numéro a étudié des relations de socialisation violente ou guerrière de petits groupes d'enfants en les interprétant comme l'effet des circonstances particulières - de Chine, d'Iran, du Mozambique ou d'ailleurs -. En conservant bien à l'esprit l'idée que la transformation des enfants en adultes est la trame où s'inscrit toute action juvénile, on peut aussi y décrypter la part d'échec de la reproduction sociale et même la métaphore du suicide de la société. C'est sans doute la conscience confuse de l'importance symbolique que représente pour tous - au niveau du sens cette réinjection de déterminants "structurels" dans les comportements acteurs sorte de passage du particulier au général - qui explique la fascination obscure qu'a toujours exercé la violence juvénile et corollairement son rejet quasi général dans les discours.

\section{NOTES}

1. Organisation de l'ONU spécialisée sur l'enfance. Son champ d'action était limité à l'Europe lors de sa création.

2. Ce discours tenu depuis la deuxième guerre mondiale, est la contrepartie de l'émergence progressive d'un espace juridique particulier qui a transformé l'enfant en sujet quasi-autonome du droit.

3. F. Khoroskhavar, «"Bassidje", auxiliaires juvéniles de la révolution iranienne ».

4. J. Andrieu, «Les gardes rouges : des rebelles sous influence ».

5. S. Mansour, «La génération de l'intifada ».

6. F. Chauvaud, « Gavroche et ses pairs : aspects de la violence politique du groupe enfantin en France au XIXe siècle », Thomas André, « Les enfants perdus de la commune».

7. Appliquée à la période de transition où l'enfant se transforme en adulte, l'adolescence est considérée comme une catégorie mixte, mélangeant l'enfance et l'âge adulte selon des dosages qu'il faudrait analyser à chaque fois, mais dans lesquels la composante physique ; et notamment la maturité sexuelle, sert d'étalon de mesure. 8. Quelle pertinence scientifique pour le concept d'enfance? 
9. C'est la définition qu'en donne A. Percheron in La socialisation politique, A. Colin, 1993, p. 14, note 2 .

10. Cela renvoie aux syllogismes et enthymèmes, soit classiques sur le sentiment maternel, l'éducation, soit contemporains sur l'enfant victime ou la disparition de l'enfance.

11. L'Enfant et la Vie Familiale sous l'Ancien Régime, Plon, 1960, réed. Seuil 1973.

12. La création de l'école gratuite et obligatoire par la IIIe République rendant possible l'entrée de la quasi totalité de chaque classe d'âge dans cet espace, n'est que le couronnement de cette évolution.

13. Voir Marc Soriano, La semaine de la comète, rapport secret sur l'enfance au XIXe, Stock 1981. Ce rapport dont l'auteur est vraisemblablement le Comte de Gobineau et le destinataire Alexis de Tocqueville, envisage les rapports jeunes/adultes en termes de guerre civile.

14. A Percheron analyse avec finesse ces deux notions. Elle montre la difficulté qu'il y a à en donner un contenu satisfaisant. Elle insiste notamment sur la dimension statistique de la notion de classe d'âge. La partition de la population en classes d'âges séquentielles à laquelle nous a habitué la multiplication des sondages et des enquêtes d'opinion et la démographie considère la vie comme un continuum qu'on peut couper selon les impératifs statistiques : égalité d'intervalles, égalité numérique de groupes. Dans ce cadre les frontières entre jeunesse et âge adulte sont mobiles, peu significatives ou effacées, selon l'objet de l'enquête.

15. "Tant que les tendances, les instincts les désirs règnent sans contrepoids, tant que notre conduite dépend uniquement de leur intensité respective, ce sont de perpétuelles sautes de vent, de brusques à coups comme ceux qui se produisent chez l'enfants ou chez le primitif, et qui, en divisant sans cesse la volonté contre elle-même, en la dispersant à tous les vents du caprice, l'empêche de se constituer avec cette unité et de se suivre avec cette persévérance qui sont les conditions primordiales de la personnalité". Emile Durkheim, L'éducation morale, Paris, PUF 1992.

16. On peut aussi rapprocher cette thèse de celle de Norbert Elias qui voit dans le processus de civilisation une maitrise progressive des pulsions, et faire ainsi de l'éducation la partie émergente de ce processus.

17. Voir sur ce point S Audouin-Rouzeau, «L'enfant héroïque en 1914-1918 » in Guerre et Cultures 1914-1918, A. Colin, 1994.

18. Métaphore par le rapport symbolique entre les personnages sculptés et la personnification de la patrie qui les domine, métonymie puisque de l'adolescent au vieillard chenu, tous ces personnages sont comme l'indique le premier strophe de la Marseillaise des "enfants de la patrie" Enfin le groupe sculpté en 1835/36 pérénnise à l'usage des générations futures un modèle de comportement patriotique.

19. D'ailleurs sur ce point le discours sur l'enfance ne fait que redoubler à son niveau particulier une évolution plus générale des idées sur la société qui exclut progressivement de la scène politique la violence confisquée par l'Etat wébérien. 20. L.J. Duclos, « Les enfants et la violence politique ».

21. A la période égocentrique, où l'enfant prend son point de vue propre pour un absolu et déforme le réel, succède vers dix/douze ans la période sociocentrique où à l'inverse, la pensée se désubjectivise, atteint un état de relativisme dans lequel l'enfant est désormais capable d'entrer dans le point de vue de ses interlocuteurs. Sur le plan affectif cela signifie que l'enfant va tendre à se détacher de son environnement le plus 
immédiat et notamment de sa famille. Un certain nombre des études de cas que nous réunissons prennent leurs exemples autour de cet âge.

22. A Peralva s'interroge sur les codes de la violence des banlieues sur leur "verbalisation" et conclue qu'on n'a pas affaire à un mouvement politique.

23. J.C. Legrand, F. Weissman, « Enfants soldats et usages de la violence au Mozambique».

24. G. Nicolas, « Participation juvénile aux violences politiques nigérianes ».

25. Mao a refermé brutalement l'espace d'autonomie des gardes rouges qu'il avait ouvert. Ce cynisme avéré a gommé de la mémoire collective l'autonomie intermédiaire qui nous intéresse ici.

26. Avec dans ce dernier cas la réapparition de petits groupes de casseurs tentant de profiter du désordre.

27. L.A. Coser, Les fonctions du conflit sociologique, PUF, 1982. Pour L. Coser conflit réaliste veut dire conflit qui n'est qu'un moyen pour atteindre un objectif et conflit irréaliste conflit qui est à lui même son propre but et son propre contenu.

28. Pour tout dire éphémère. Ephémère parce que liées à des événements ou à une conjoncture courte (révolution culturelle, guerre Iran-Irak...), éphémère car les jeunes s'évaporent mystérieusement du champ de la violence, sont tués ou deviennent vite adultes. Nous touchons là à une contrainte structurelle qui explique la fragilité des formes de la violence juvénile ainsi que leur renouvellement permanent.

29. Ces observations doivent d'ailleurs être nuancées. Sur le plan psychologique L.J. Duclos remarque à juste titre que ces sentiments ou ces attitudes sont en partie partagés avec les jeunes adultes, notamment les jeunes militaires. En réalité la violence infantile ne désigne pas un contenu mais qualifie un comportement dont les jeunes adultes ne sont pas bien éloignés et auxquels ils sont toujours, au moins par archaïsme, susceptibles de retourner. La psychologie apparait ici, plus comme un dénominateur commun que comme une originalité.

30. "Si tu les regardes, tu n'observeras aucun élément qui serait commun à tous, mais tu verras des ressemblances, des airs de famille en grand nombre", cité par R Boudon, L'art de se persuader, Fayard, 1992, p. 325.

31. Ce rapport englobé/englobant, ou partie/tout correspond à une relation synecdochique entre l'ensemble réduit de la violence juvénile et l'ensemble plus vaste constitué par la jeunesse.

\section{RÉSUMÉS}

La jeunesse, catégorie construite à l'aide d'une relation en général parent/enfant ne définit pas une classe ou un groupe social, mais un support logique à des discours généraux. Cette attitude rhétorique impose implicitement à l'analyse politique un cadre à large focale embrassant toute la société et favorise ainsi une captation unidimensionnelle où le sens de la violence enfantine est réduit à l'instrumentalisation ou à la manipulation des enfants par les adultes. Il faut au contraire interpréter les situations de socialisation violentes et guerrières des groupes d'enfants comme le produit des circonstances particulières (Chine, Iran, Mozambique...). La transformation des 
enfants en adultes est la trame où s'inscrit toute action juvénile, on peut aussi y décrypter la part d'échec de la reproduction sociale et même la métaphore du suicide de la société. C'est sans doute la conscience confuse de l'importance symbolique que représente pour tous - au niveau du sens - cette réinjection de déterminants «structurels » dans les comportements acteurs - sorte de passage du particulier au général - qui explique la fascination obscure qu'a toujours exercé la violence juvénile et corollairement son rejet quasi général dans les discours.

\section{INDEX}

Mots-clés : enfants, violence 